\title{
Electrophysiological determinant for induction of isthmus dependent counterclockwise and clockwise atrial flutter in humans
}

\author{
J-L Lin, L-P Lai, L-J Lin, Y-Z Tseng, W-P Lien, S K S Huang
}

\begin{abstract}
Objective-To investigate the electrophysiological determinant underlying the electrical induction of counterclockwise and clockwise isthmus dependent atrial flutter.

Patients and methods-The isthmus bordered by the inferior vena caval orificetricuspid annulus-coronary sinus ostium (IVCO-TA-CSO) has been assumed to be the site of both slow conduction and unidirectional block critical to the initiation of atrial flutter. Trans-isthmus and the global atrial conduction were studied in 25 patients with isthmus dependent atrial flutter (group A) and in 21 patients without atrial flutter (group B), by pacing at the coronary sinus ostium and the low lateral right atrium (LLRA) and mapping with a 20 pole Halo catheter in the right atrium.
\end{abstract}

Results-Mean (SD) fluoroscopic isthmus length between the coronary sinus ostium and LLRA sites was $28.1(4.0) \mathrm{mm}$ in group $A$ and 28.0 (3.9) $\mathrm{mm}$ in group $B$ $(p=0.95)$, but the trans-isthmus conduction velocity of both directions at various pacing cycle lengths was nearly halved in group A compared with group B (mean $0.39-0.46 \mathrm{~m} / \mathrm{s} v 0.83-0.89 \mathrm{~m} / \mathrm{s}, \mathrm{p}<0.0001)$. Pacing at coronary sinus ostium directly induced counterclockwise atrial flutter in 14 patients and pacing at LLRA induced clockwise atrial flutter in 11 patients, following abrupt unidirectional transisthmus block. Transient atrial tachyarrhythmias preceded the onset of atrial flutter in 10 counterclockwise and six clockwise cases of atrial flutter. None of the group $B$ patients had inducible atrial flutter even in the presence of transisthmus block. The intra- and interatrial conduction times, as well as the conduction velocities at the right atrial free wall and the septum, were similar and largely within the normal range in both groups. Conclusions-Critical slowing of the trans-IVCO-TA-CSO isthmus conduction, but not the unidirectional block or the global atrial performance, is the electrophysiological determinant of the induction of counterclockwise and clockwise isthmus dependent atrial flutter in man. (Heart 1999;81:73-81)

Keywords: atrial flutter; electrical induction; radiofrequency catheter ablation
Our knowledge of human atrial flutter has progressively evolved, ${ }^{1-3}$ particularly with regard to the delineation of the reentry circuit and the consequent success in radiofrequency catheter ablation. Several studies ${ }^{4-6}$ incorporating circumferential intra-atrial mapping, entrainment, and intracardiac echocardiography have established that the reentry circuit of either counterclockwise or clockwise isthmus dependent atrial flutter in man is large and confined to the right atrium. The atrial activation wavefront circulates between the anterior free wall and the septum, using the narrow isthmus bordered by the inferior vena caval orifice (IVCO), the tricuspid annulus (TA), and the coronary sinus ostium (CSO) as an obligatory conduction path. Complete interruption of the conduction across the IVCOTA-CSO isthmus results in the abolition of all isthmus dependent atrial flutter. ${ }^{378}$ Despite the clarification of the reentry circuit, however, some questions remain about the electrophysiological determinants underlying the electrical induction of isthmus dependent atrial flutter in susceptible patients.

Because of the existence of multiple verges and orifices, the low right atrium is a structure for potential anisotropism. Clinical studies ${ }^{10}$ have strongly suggested a discrete area of slow conduction in the inferior-posterior right atrium, most probably between the coronary sinus ostium and the low lateral right atrium (LLRA), because of the demonstration of entrainment by pacing at the coronary sinus ostium and LLRA. Taking account of the unique nature of the local electrical and anatomical features, we hypothesise that the atrial isthmus bordered by the inferior vena caval orifice, the tricuspid annulus, and the coronary sinus ostium in the low right atrium is the crucial site of slow conduction and unidirectional block, and determines the electrophysiological inducibility of both counterclockwise and clockwise isthmus dependent atrial flutter. To test this hypothesis, we compared the trans-isthmus conduction velocity and atrial conduction property in patients with and without inducible isthmus dependent atrial flutter by direct pacing and measurement across the IVCO-TA-CSO isthmus in the low right atrium.

\section{Methods}

PATIENT CHARACTERISTICS

Between April 1996 and February 1997, we studied 25 consecutive patients with clinical atrial flutter or flutter inducible by programmed electrical stimulation (group A) and 21 patients without atrial flutter (group B). 


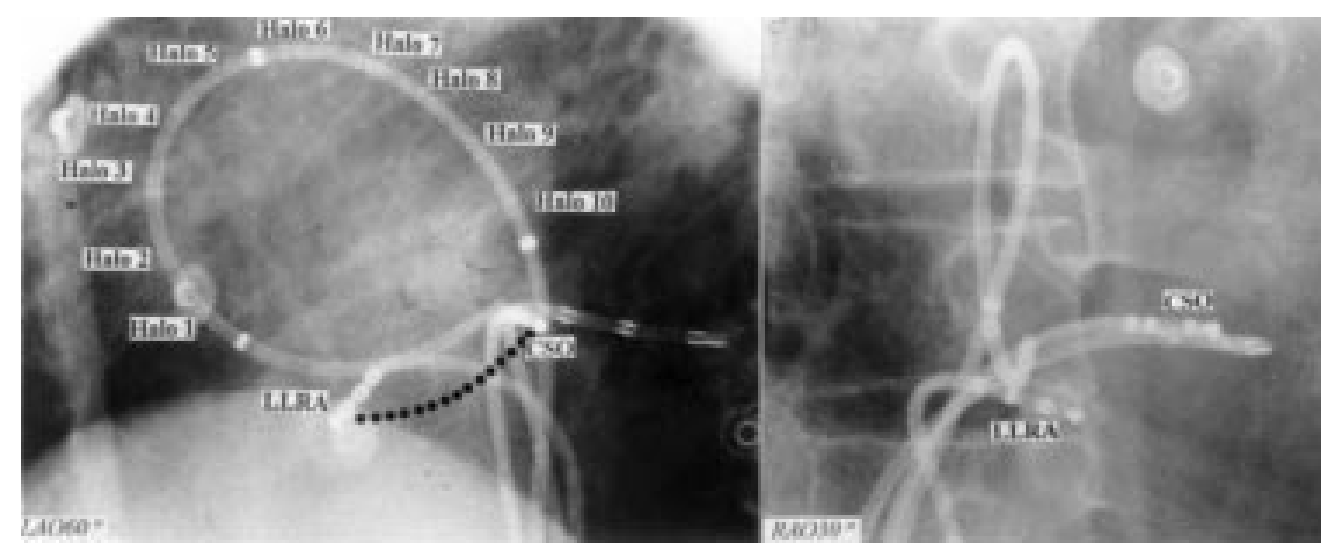

Figure 1 Orthogonal fluoroscopic views showing the positions of the 20 pole Halo catheter and the two pacing electrodes at the low lateral right atrium (LLRA) and coronary sinus ostium (CSO). On the left is the $60^{\circ}$ left anterior oblique (LAO) view, and on the right is the $30^{\circ}$ right anterior oblique (RAO) view. As shown, the Halo 1 to Halo 5 bipoles are to map the anterolateral free wall of right atrium, while Halo 6 to Halo 10 bipoles map the septum. Another coronary sinus catheter is in the position for guiding the location of the coronary sinus ostium and for mapping the interatrial conduction. The isthmus length (the dotted line between the tips of the two deflectable catheters at the LLRA and CSO) in the IVCO-TA-CSO region was $28 \mathrm{~mm}$ in the $60^{\circ} \mathrm{LAO}$ view.

In the 25 group A patients, there were 17 men and eight women, mean (SD) age 54 (17) years (range 20 to 74 years). The clinical episodes of atrial flutter were documented in 22 patients and later all were confirmed to be isthmus dependent. The right atrial activation pattern of atrial flutter was counterclockwise oriented in 18 patients, clockwise in two, and both directions in two. The remaining three patients had inducible isthmus dependent atrial flutter during electrophysiological studies for the evaluation of other tachycardias, which were atrioventricular nodal reentrant tachycardia (two patients) and atrioventricular reentrant tachycardia incorporating a concealed left lateral accessory pathway (one patient).

Associated medical diseases were hypertension (3), coronary artery disease after bypass grafting surgery (1), aortic valve replacement for aortic regurgitation (1), mild Ebstein anomaly (1), and chronic obstructive pulmonary disease (1).

In the 21 group $B$ patients, there were 16 men and five women, mean (SD) age 52 (15) years (range 22 to 77 years). Their clinical arrhythmias were Wolff-Parkinson-White syndrome (3), atrioventricular reentrant tachycardia incorporating a concealed accessory pathway (7), atrioventricular nodal reentrant tachycardia (7), atrial tachycardia (3), and sick sinus syndrome (1). Associated medical diseases were hypertension (3), coronary artery disease (1), and chronic obstructive pulmonary disease (1).

\section{ELECTROPHYSIOLOGICAL STUDY AND}

RADIOFREQUENCY CATHETER ABLATION

All patients were studied in the postabsorptive state. Antiarrhythmic drugs were discontinued for at least 5 half lives. A duodecapolar Halo catheter (Cordis-Webster, Baldwin Park, California, USA) was introduced to the right atrium through a femoral vein and manipulated to be parallel with the tricuspid annulus. The first five bipole pairs (Halo 1 to 5 , fig 1 ) were positioned to map the electrical activities in the anterolateral free wall of the right atrium, while the other five bipoles (Halo 6 to 10) in the interatrial septum.

Two deflectable quadripolar catheters (Mansfield EP, Boston Scientific, Watertown, Massachusetts, USA) with a $2 \mathrm{~mm}$ distal bipole spacing were positioned at the LLRA and the coronary sinus ostium to bracket the IVCOTA-CSO isthmus. The LLRA site was fluoroscopically selected near the junction of the lateral right atrium and the IVCO. Repeated injections of contrast medium to the inferior vena cava and the coronary sinus were performed to identify the coronary sinus ostium, the right atrial floor, and the final catheter location. Additional reference electrodes were positioned at the His bundle region and in the distal coronary sinus. Care was taken to keep the Halo catheter and the pacing electrode catheters on the same positions throughout the study.

All the patients were studied in sinus rhythm before attempted radiofrequency catheter ablation of clinical tachyarrhythmias. Clinical atrial flutter was terminated in advance by atrial overdrive pacing in three patients and by low energy direct current cardioversion in one. Incremental pacing up to the loss of 1:1 atrial capture was performed at the LLRA and the coronary sinus ostium to induce atrial flutter. Meanwhile, an entrainment study was performed by pacing at the LLRA and coronary sinus ostium. ${ }^{11}{ }^{12}$ Atrial local electrograms were recorded simultaneously from 13 right atrial sites (10 from the Halo catheter, two from the LLRA and CSO sites across the isthmus, one from the His bundle region) and three left atrial sites (from the coronary sinus) to demonstrate the intra-atrial and interatrial activation sequences during pacing and during the induction of atrial flutter.

All intracardiac electrograms were filtered between 30 and $500 \mathrm{~Hz}$ and displayed on a 12 lead surface ECG by a computerised electrophysiology recording system equipped with optical disk data storage (Cardiolab, Prucker Engineering Inc, Houston, Texas, USA). The mapping data could be monitored and traced 
A

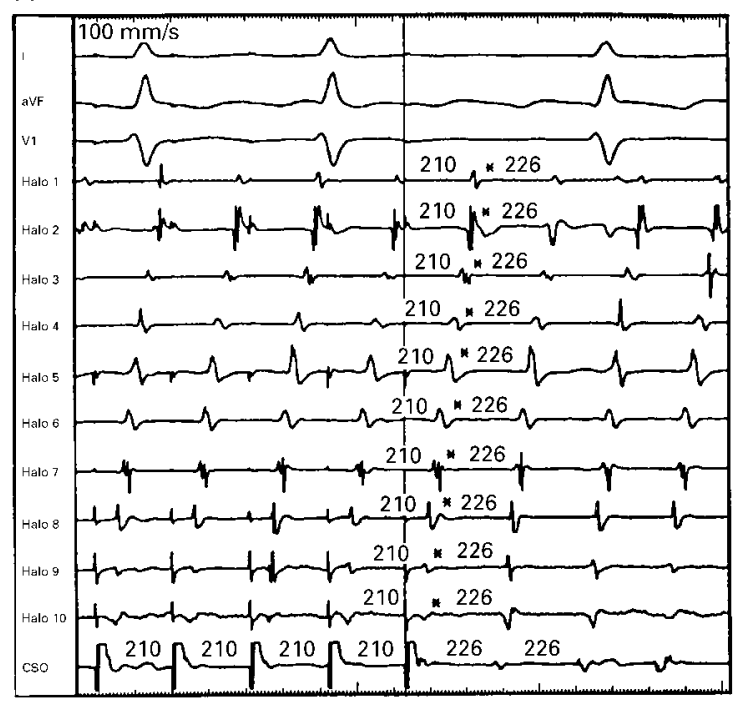

B

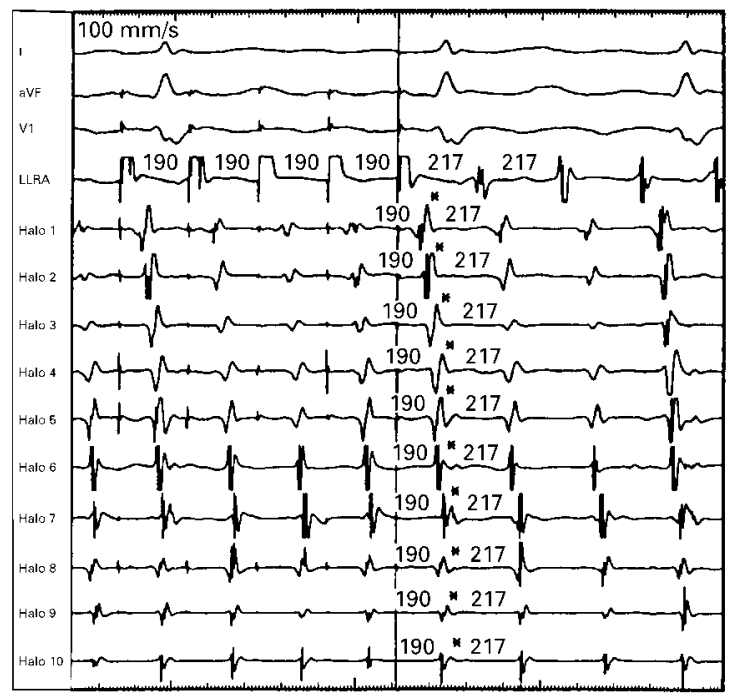

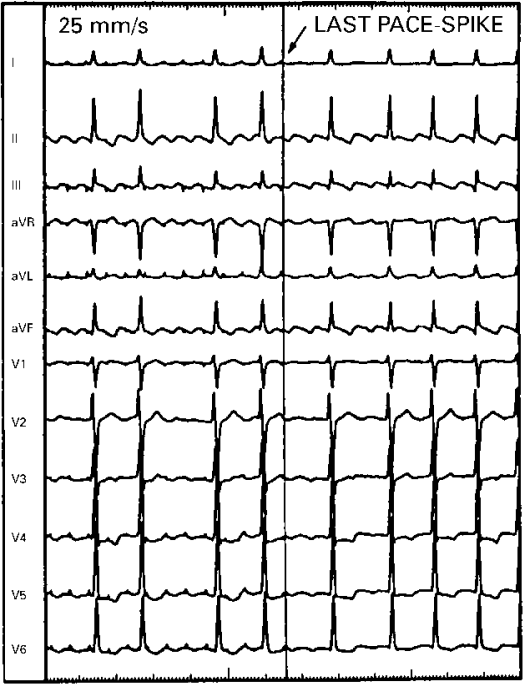

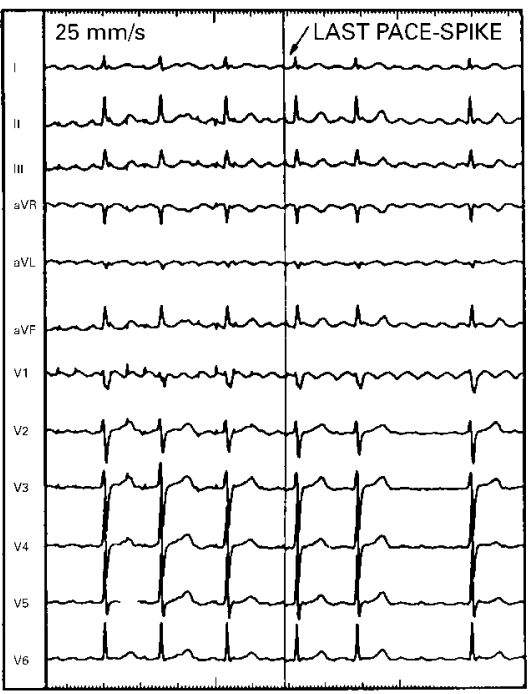

Figure 2 Demonstration of entrainment with concealed fusion by pacing at the coronary sinus ostium (CSO) for counterclockwise atrial flutter $(A)$ and by pacing at the LLRA for clockwise atrial futter $(B)$. Note the mainly negative flutter wave in the inferior ECG leads for counterclockwise atrial flutter and the mainly positive flutter wave for clockwise atrial flutter. Neither the surface ECG nor the intra-atrial activation sequence in $(A)$ and $(B)$ show evidence of fusion or wavefront collision. The demonstration of entrainment with concealed fusion indicates the collision or block of the antidromic pacing wavefront inside the slow conduction zone of the atrial flutter circuit. The short stimulus to onset of the ECG flutter wave interval in both situations indicates that the coronary sinus ostium was the exit of the slow conduction zone in counterclockwise atrial flutter and the low lateral right atrium was the exit in clockwise atrial flutter. The onset of the flutter wave was recognised as the beginning of the downward deflection of the saw tooth morphology of the flutter wave since the IVCO-TA-CSO isthmus is located in the low right atrium. The cycle length of the counterclockwise atrial flutter $(A)$ was $226 \mathrm{~ms}$, and that of the clockwise atrial flutter (B) was $217 \mathrm{~ms}$. The asterisk indicates the last entrained beat.

for real time activation sequence analysis, or laser printed for future review. The atrial pacing was delivered by a digital stimulator (Bloom Associates, Reading, Pennsylvania, USA) at two to five times the diastolic threshold and with a pulse duration of $2 \mathrm{~ms}$.

Cross sectional and $M$ mode echocardiography with colour flow mapping was performed at baseline to record the cardiac chamber dimensions, cardiac performance, and intracardiac flow.

For radiofrequency catheter ablation of the isthmus dependent atrial flutter, a linear lesion was created between the tricuspid annulus and inferior vena cava by a deflectable ablation catheter with a $4 \mathrm{~mm}$ or $10 \mathrm{~mm}$ distal electrode and a tip thermistor (Dr Osypka $\mathrm{GmbH}$, Grenzach-Wyhlen, Germany). Unmodulated
$500 \mathrm{kHz}$ radiofrequency current (HAT 300S, Dr Osypka $\mathrm{GmbH}$ ) was applied in the temperature control mode with a target temperature set at $60^{\circ} \mathrm{C}$. The end point of ablation was bidirectional conduction block across the IVCO-TA-CSO isthmus as revealed by pacing at either side of the isthmus.

DEFINITIONS AND MEASUREMENTS

Isthmus dependent atrial flutter is defined by the demonstration of both entrainment at the isthmus region and successful radiofrequency catheter ablation targeted at the isthmus. Atrial flutter can be either counterclockwise activation (fig 2A) or clockwise activation (fig 2B) in the right atrium. By pacing at the coronary sinus ostium during counterclockwise atrial flutter, and at the LLRA during clockwise atrial 
Table 1 Comparison of global atrial conduction characteristics between 25 patients with isthmus dependent atrial flutter (group A) and 21 patients without (group B)

\begin{tabular}{|c|c|c|c|c|}
\hline \multicolumn{2}{|c|}{ Global RA function } & Group $A$ & Group B & $p$ Value \\
\hline \multicolumn{5}{|c|}{ Antegrade } \\
\hline \multicolumn{2}{|c|}{$\mathrm{P}$ wave duration $(\mathrm{ms})$} & $119.3(17.5)$ & $114.4(11.7)$ & 0.25 \\
\hline \multicolumn{2}{|c|}{$\mathrm{P}$ wave amplitude (mV) } & $0.20(0.06)$ & $0.18(0.04)$ & 0.28 \\
\hline \multicolumn{2}{|c|}{ P-HBE (ms) } & $34.1(7.5)$ & $32.8(10.5)$ & 0.20 \\
\hline \multicolumn{2}{|l|}{$\mathrm{P}-\mathrm{CSO}(\mathrm{ms})$} & $60.5(17.2)$ & $54.6(10.6)$ & 0.63 \\
\hline \multicolumn{2}{|l|}{ P-DCS (ms) } & $81.0(22.3)$ & $75.1(13.3)$ & 0.29 \\
\hline \multicolumn{5}{|c|}{ Retrograde } \\
\hline \multicolumn{5}{|c|}{ RA free wall conduction velocity $(\mathrm{m} / \mathrm{s})$} \\
\hline \multirow[t]{5}{*}{ LLRA PCL } & $500 \mathrm{~ms}$ & $0.98(0.09)$ & $1.00(0.13)$ & 0.07 \\
\hline & $400 \mathrm{~ms}$ & $0.97(0.11)$ & $1.00(0.10)$ & 0.66 \\
\hline & $300 \mathrm{~ms}$ & $0.96(0.12)$ & $0.98(0.10)$ & 0.55 \\
\hline & $250 \mathrm{~ms}$ & $0.95(0.10)$ & $0.97(0.10)$ & 0.51 \\
\hline & $200 \mathrm{~ms}$ & $0.91(0.11)$ & $0.94(0.07)$ & 0.29 \\
\hline \multicolumn{5}{|c|}{ RA septum conduction velocity $(\mathrm{m} / \mathrm{s})$} \\
\hline \multirow[t]{5}{*}{ CSO PCL } & $500 \mathrm{~ms}$ & $0.91(0.09)$ & $0.98(0.15)$ & 0.054 \\
\hline & $400 \mathrm{~ms}$ & $0.90(0.09)$ & $0.99(0.16)$ & 0.020 \\
\hline & $300 \mathrm{~ms}$ & $0.91(0.10)$ & $0.97(0.14)$ & 0.094 \\
\hline & $250 \mathrm{~ms}$ & $0.90(0.10)$ & $0.97(0.12)$ & 0.034 \\
\hline & $200 \mathrm{~ms}$ & $0.86(0.09)$ & $0.96(0.17)$ & 0.014 \\
\hline
\end{tabular}

Data are mean (SD)

ANOVA comparisons of conduction velocities at various pacing cycle lengths in the right atrial (RA) free wall and the RA septum were non-significant in group A and group B patients.

CSO, coronary sinus ostium; DCS, distal coronary sinus; HBE, His bundle electrogram; LLRA, low lateral right atrium; PCL, pacing cycle length.

flutter, we could demonstrate entrainment with concealed fusion and a very short $(<20 \mathrm{~ms})$ interval from the stimulus to the onset of the flutter wave (fig 2A and 2B). The onset of the flutter wave was taken at the beginning of the downward component of the saw tooth flutter wave in inferior ECG leads. Isthmus dependent atrial flutter is regarded synonymous with typical atrial flutter, as suggested by Lesh and Kalman. ${ }^{13}$

Trans-isthmus conduction time is considered as the activation time across the atrial isthmus. In the present study, the conduction time was calculated by pacing directly at either the coronary sinus ostium or the LLRA and measuring at the opposite end of the isthmus. The activation interval was taken from the pace spike at one side of the isthmus to the onset of the local electrogram of the distal bipoles at the other side. The isthmus length was the curve distance between the distal electrodes of the coronary sinus ostium and the LLRA pacing catheters, determined by fluoroscopic imaging of the right atrial floor in the $60^{\circ}$ left anterior oblique view (fig 1). The trans-isthmus conduction velocity could then be estimated by the fluoroscopic isthmus length divided by the trans-isthmus conduction time.

Antegrade atrial conduction property was estimated in sinus rhythm by the maximum $P$ wave duration on the 12 lead ECG, the P wave amplitude at ECG lead II, and the onset of P wave to local atrial activation at the His bundle region, the coronary sinus ostium, and the distal coronary sinus. ${ }^{14} 15$

Retrograde atrial conduction property was evaluated by the atrial conduction time along the anterior free wall of right atrium (Halo 1 to 5, fig 1) during LLRA pacing and along the interatrial septum (Halo 10 to 6, fig 1) during coronary sinus ostium pacing. Atrial conduction velocity in the free wall and the septum of the right atrium could be estimated by the activated Halo electrode distance divided by the conduction time.
All the local atrial activation times were measured at the onset of the first $45^{\circ}$ slope on the local electrograms.

\section{STATISTICS}

Continuous data were expressed as mean (SD), and compared by unpaired Student's $t$ test between the two groups and analysis of variance (ANOVA) among five different pacing cycle lengths. Categorical data were compared by the $\chi^{2}$ test with Yates's correction. A $\mathrm{p}$ value less than 0.05 was considered as significant.

\section{Results}

GLOBAL ATRIAL CONDUCTION

As shown in table 1, the antegrade intra-atrial and interatrial conduction characteristics measured by the $\mathrm{P}$ wave duration, the $\mathrm{P}$ wave amplitude, and the $\mathrm{P}$ wave onset to local atrial activation at the His bundle region, the coronary sinus ostium, and the distal coronary sinus were similar in group A and group B patients. The $\mathrm{P}$ wave duration on the 12 lead ECG was more than $120 \mathrm{~ms}$ in nine patients in each group with and without atrial flutter (9/25 v $9 / 21, p=0.86)$. The $P$ wave amplitude in lead II of the ECG was more than $0.3 \mathrm{mV}$ in three patients in group A, but in none in group B $(p=0.73)$. On the other hand, the conduction velocity of the retrograde right atrium free wall measured by LLRA pacing was similar and within the normal limit in both groups (mean range from 0.91 to $1.0 \mathrm{~m} / \mathrm{s}$, table 1 ). However, the conduction velocity of the right atrial septal wall measured by coronary sinus ostium pacing was slightly slower in group A than in group B $(0.86-0.91 \mathrm{~m} / \mathrm{s} v 0.96-0.99 \mathrm{~m} / \mathrm{s}, \mathrm{p}<0.05)$, although all the data were within the normal range. The right atrial conduction in both the free wall and the septum was non-decremental.

In the 25 group A patients, echocardiographic studies showed left atrial enlargement in three and right atrial enlargement in two, whereas in the 21 group B patients, three had left atrial enlargement and none had right atrial enlargement. Left ventricular hypertrophy was noted in two patients in group A and one in group B. Mild tricuspid regurgitation (from Ebstein anomaly) was noted in one patient in group A. All group A and group B patients had a normal systolic function of both left and right ventricles.

TRANS-ISTHMUS CONDUCTION

In contrast to the global atrial function, the trans-isthmus conduction time in either direction was much longer at all pacing cycle lengths in group A (mean range from 62.1 to $74.7 \mathrm{~ms}$ ) than in group B (mean range from 33 to $35 \mathrm{~ms}$, $\mathrm{p}<0.0001$ ) (table 2), while the measured isthmus length was similar in the two groups (28.1 (4.0) $\mathrm{mm}$ v 28.0 (3.9) $\mathrm{mm}, \mathrm{p}=0.95)$. Therefore the bidirectional conduction velocity across the IVCO-TA-CSO isthmus at all pacing cycle lengths was nearly halved in group A compared with group B $(0.39-0.46 \mathrm{~m} / \mathrm{s} v$ $0.83-0.89 \mathrm{~m} / \mathrm{s}, \mathrm{p}<0.0001$ ) (table 2). The impulse propagation across the isthmus in group A patients was mildly decremental when pacing cycle length varied from $500 \mathrm{~ms}$ to 200 
Table 2 Comparison of trans-isthmus conduction between the 25 patients with isthmus dependent atrial flutter (group A) and the 21 patients without (group B)

\begin{tabular}{|c|c|c|c|c|c|c|c|}
\hline \multirow{2}{*}{\multicolumn{2}{|c|}{ Trans-isthmus conduction }} & \multicolumn{3}{|c|}{ Conduction time (ms) } & \multicolumn{3}{|c|}{ Conduction velocity $(\mathrm{m} / \mathrm{s})$} \\
\hline & & Group $A$ & Group B & $p$ Value & Group $A$ & Group B & $p$ Value \\
\hline \multicolumn{8}{|c|}{ LLRA to CSO direction } \\
\hline \multirow[t]{5}{*}{ LLRA PCL } & $500 \mathrm{~ms}$ & $63.2(8.6)$ & $33.0(6.8)$ & $<0.0001$ & $0.45(0.07)$ & $0.88(0.21)$ & $<0.0001$ \\
\hline & $400 \mathrm{~ms}$ & $62.1(9.6)$ & $33.0(6.8)$ & $<0.0001$ & $0.46(0.07)$ & $0.89(0.22)$ & $<0.0001$ \\
\hline & $300 \mathrm{~ms}$ & $63.5(9.6)$ & $34.0(7.4)$ & $<0.0001$ & $0.45(0.07)$ & $0.87(0.21)$ & $<0.0001$ \\
\hline & $250 \mathrm{~ms}$ & $65.4(10.5)$ & $33.0(8.6)$ & $<0.0001$ & $0.44(0.07)$ & $0.89(0.22)$ & $<0.0001$ \\
\hline & $200 \mathrm{~ms}$ & $71.5(11.8)$ & $34.0(8.5)$ & $<0.0001$ & $0.40(0.06)$ & $0.86(0.20)$ & $<0.0001$ \\
\hline \multicolumn{8}{|c|}{ CSO to LLRA direction } \\
\hline \multirow[t]{5}{*}{ CSO PCL } & $500 \mathrm{~ms}$ & $65.9(11.1)$ & $33.0(6.3)$ & $<0.0001$ & $0.43(0.07)$ & $0.87(0.18)$ & $<0.0001$ \\
\hline & $400 \mathrm{~ms}$ & $66.8(12.2)$ & $34.1(6.8)$ & $<0.0001$ & $0.43(0.07)$ & $0.85(0.18)$ & $<0.0001$ \\
\hline & $300 \mathrm{~ms}$ & $68.0(12.7)$ & $33.0(6.8)$ & $<0.0001$ & $0.42(0.08)$ & $0.88(0.21)$ & $<0.0001$ \\
\hline & $250 \mathrm{~ms}$ & $68.8(13.6)$ & $34.0(6.8)$ & $<0.0001$ & $0.42(0.07)$ & $0.87(0.20)$ & $<0.0001$ \\
\hline & $200 \mathrm{~ms}$ & $74.7(16.5)$ & $35.0(6.5)$ & $<0.0001$ & $0.39(0.08)$ & $0.83(0.16)$ & $<0.0001$ \\
\hline \multicolumn{2}{|c|}{ Isthmus length (mm) } & $28.1(4.0)$ & $28.0(3.9)$ & 0.95 & & & \\
\hline
\end{tabular}

Data are mean $(\mathrm{SD})$.

ANOvA comparison of trans-isthmus conduction velocities at various PCLs was significant in group A patients in LLRA to CSO direction ( $\mathrm{p}<0.03$, ANOVA), but not CSO to LLRA direction $(\mathrm{p}=0.2)$, nor in either direction for group $\mathrm{B}$ patients $(\mathrm{p}=0.98$ and $\mathrm{p}$ $=0.85$, respectively).

CSO, coronary sinus ostium; LLRA, low left lateral atrium; PCL, pacing cycle length.

ms and only in the LLRA to CSO direction $(\mathrm{p}<0.03$, ANOvA). This was not observed in group B patients.

The right atrial activation sequence during either LLRA or CSO pacing in sinus rhythm in group B showed impulse wavefront collision at the Halo 5 or Halo 6 position by Halo catheter mapping (fig 3), whereas in group A, the collision wavefronts shifted to Halo 7 or 8 during LLRA pacing and to Halo 3 or 4 during coronary sinus ostium pacing due to the slow transisthmus conduction (fig 3).

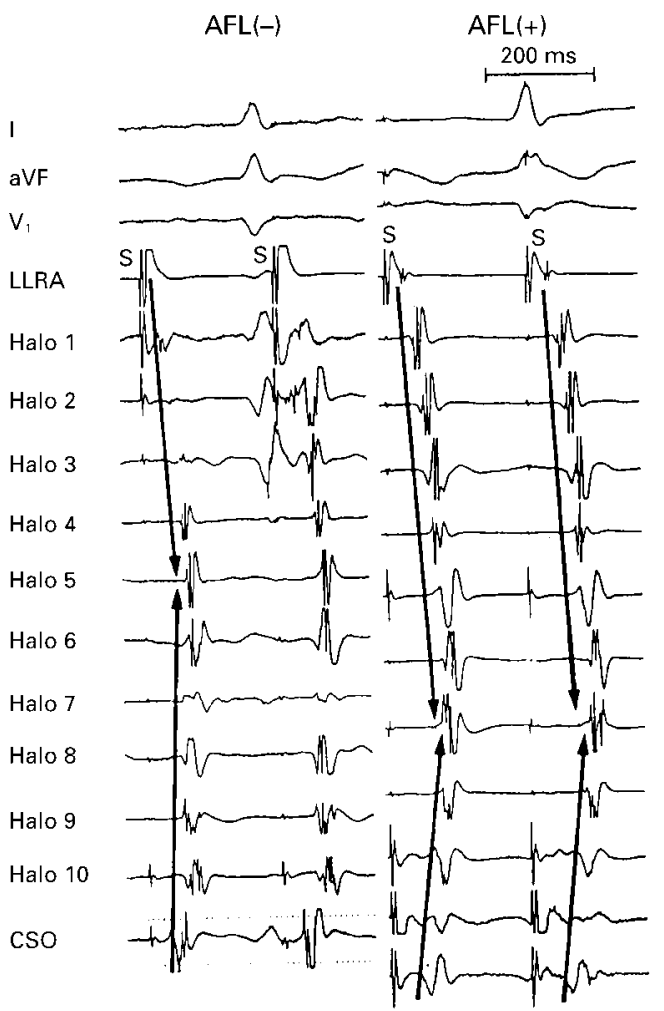

ELECTRICAL INDUCTION OF ISTHMUS DEPENDENT ATRIAL FLUTTER

Direct coronary sinus ostium pacing at cycle length of 205 (16) $\mathrm{ms}$ (range 180 to $240 \mathrm{~ms}$ ) resulted in abrupt coronary sinus ostium to LLRA trans-isthmus conduction block and the initiation of counterclockwise isthmus dependent atrial flutter in $14(56 \%)$ of the 25 group A patients (fig 4). On the other hand, direct LLRA pacing at cycle length of 199 (19) ms (range 170 to $250 \mathrm{~ms}$ ) caused a similar LLRA to coronary sinus ostium trans-isthmus block

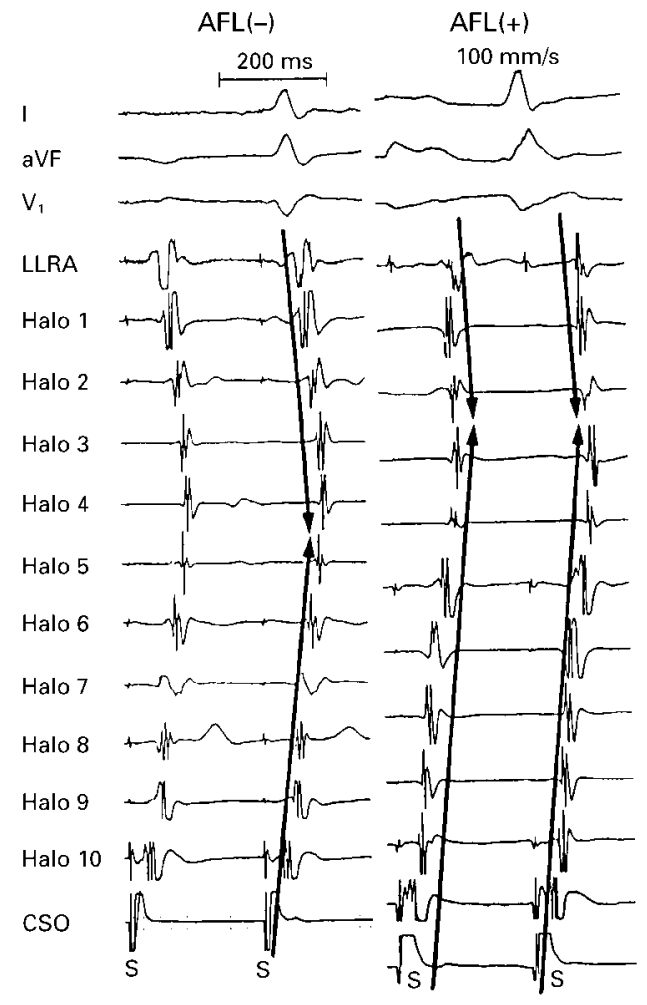

Figure 3 The retrograde right atrial $(R A)$ activation sequence of Halo catheter mapping by low lateral right atrium (LLRA) pacing (left two panels) and coronary sinus ostium (CSO) pacing (right two panels) in sinus rhythm in a patient with atrial flutter (atrial flutter + ) and a patient without atrial flutter (atrial flutter -). Note that in the patient without atrial flutter, the collision site of the RA free wall and RA septal wavefronts is at Halo 5. However, in the patient with atrial flutter, the collision site shifts to Halo 7 during LLRA pacing and to Halo 3 during coronary sinus ostium pacing. S, pacing spike. The pacing cycle length was $250 \mathrm{~ms}$, and the paper speed was $100 \mathrm{~mm} / \mathrm{s}$ in all four panels. 


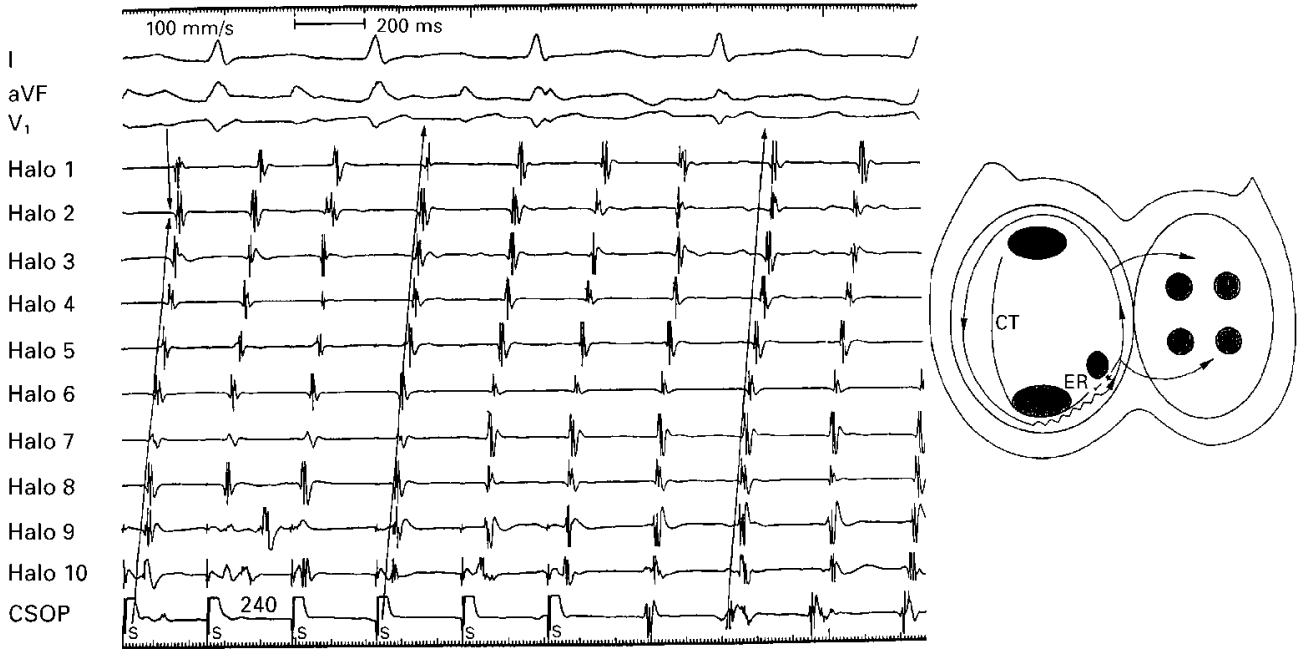

Figure 4 Induction of counterclockwise atrial flutter by pacing at the coronary sinus ostium site at the cycle length of 240 ms. Note the abrupt unidirectional block across the isthmus (between Halo 1 and 10) and the initiation of the counterclockwise atrial flutter (cycle length $244 \mathrm{~ms}$ ), after repenetration of the right atrial wavefront from the low lateral right atrial end to the coronary sinus ostium end of the isthmus. (See diagram.) CSOP, coronary sinus ostium pacing; CT, crista terminalis; ER, eustachian ridge; $S$, pacing spike. The asterisk indicates the pacing site.

and the initiation of clockwise isthmus dependent atrial flutter in 11 patients $(11 / 25,44 \%)$ (fig 5). The site of unidirectional block was never noted elsewhere along the right atrial circumference mapped by Halo catheter before the induction of isthmus dependent atrial flutter. In the 21 group B patients, unidirectional trans-isthmus block could be shown in two patients by coronary sinus ostium pacing $(2 / 21$ $v 14 / 25, \mathrm{p}<0.003$ ) and in two patients by LLRA pacing $(2 / 21 v 11 / 25, \mathrm{p}<0.01)$. However, neither the counterclockwise retrograde activation wavefront by CSO pacing nor the clockwise wavefront by LLRA pacing could repenetrate through the isthmus region, which in turn resulted in failure of atrial flutter induction. Therefore the induction of isthmus dependent atrial flutter by CSO or LLRA pacing frequently followed the sequence of unidirectional trans-isthmus block, retrograde counterclockwise or clockwise right atrial activation and repenetration of the IVCO-TA-CSO isth- mus with a slow conduction property. However, other than the typical induction pattern, 10 distinct counterclockwise and six clockwise isthmus dependent atrial flutter episodes could be induced only after induction of a transient period of atrial tachycardia $(n=3)$ or fibrillation $(n=8)$ (fig 6 ) or following the atrial overdrive pacing for a counterpart atrial flutter $(\mathrm{n}=5)$.

The mean cycle length of the 24 counterclockwise isthmus dependent atrial flutter episodes was 223 (22) ms (range 180 to $258 \mathrm{~ms}$ ), with an LLRA to CSO trans-isthmus conduction time of 67.0 (9.5) $\mathrm{ms}$ (range 54 to $90 \mathrm{~ms}$ ), whereas the cycle length of 17 clockwise isthmus dependent episodes was 224.1 (22.4) ms (range 183 to $268 \mathrm{~ms}$ ), with a CSO to LLRA trans-isthmus conduction time of 68.6 (10.8) ms (range 54 to $94 \mathrm{~ms}$ ). The transisthmus conduction times during induced isthmus dependent counterclockwise and clockwise atrial flutter were comparable with that

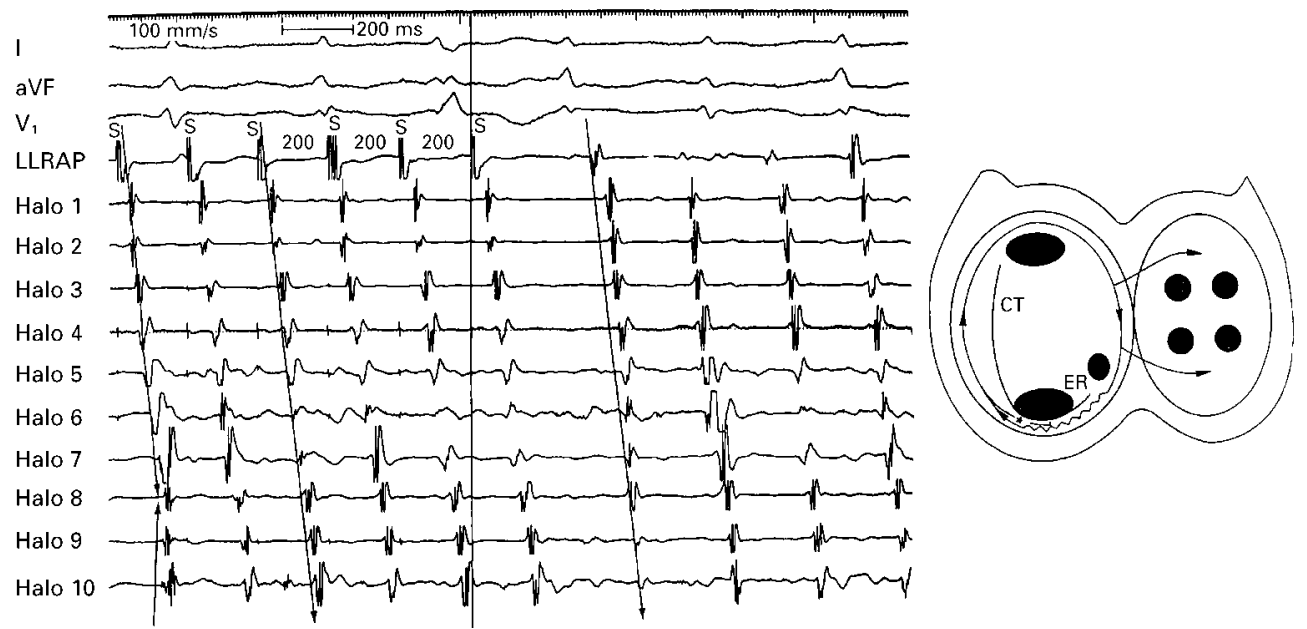

Figure 5 Induction of clockwise atrial flutter by pacing at the low lateral right atrial (LLRA) site at the cycle length of $200 \mathrm{~ms}$. Note the abrupt unidirectional block between Halo 1 and Halo 10 (that is, the isthmus zone) and the initiation of clockwise atrial flutter (cycle length $224 \mathrm{~ms}$ ) after repenetration of the right atrial wavefront from the coronary sinus ostium end to the LLRA end of the isthmus. (See diagram.) CT, crista terminalis; ER, eustachian ridge; LLRAP, LLRA pacing; S, pacing spike. 


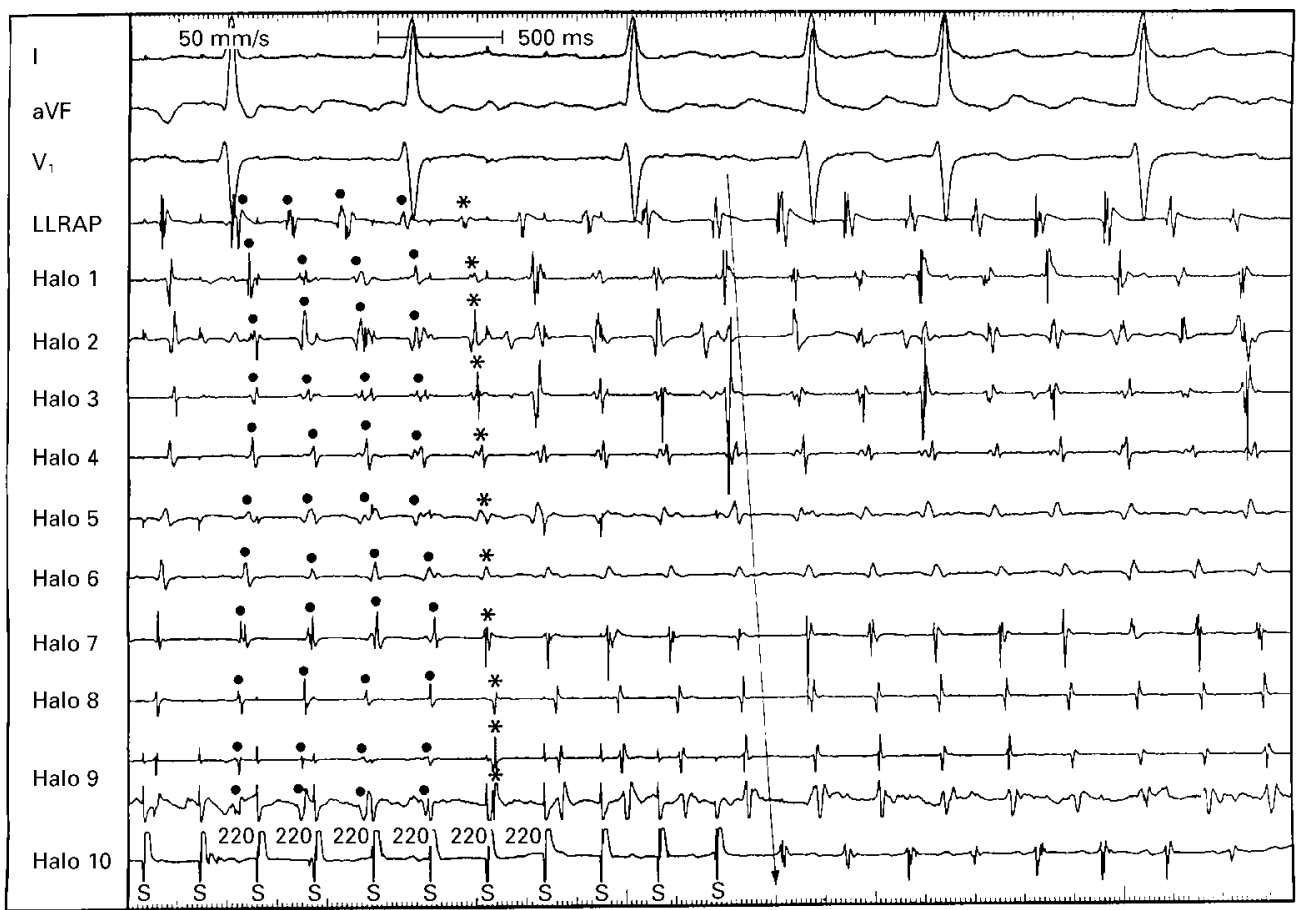

Figure 6 Induction of clockwise atrial flutter by pacing from the coronary sinus ostium site after a transient episode of multifocal atrial premature beats (indicated by dots). The pacing cycle length was $220 \mathrm{~ms}$. The cycle length of clockwise atrial flutter was 266 ms. The asterisks indicate the first beat of the sustained clockwise atrial flutter.

predicted by the corresponding LLRA and coronary sinus ostium pacing studies (table 2). The trans-isthmus conduction time constituted 30.4 (4.2) \% (range $20.9 \%$ to $38.1 \%$ ) of the cycle length of the counterclockwise atrial flutter and 30.6 (3.9)\% (range $25.2 \%$ to 38.7 $\%)$ of the clockwise type. All counterclockwise and clockwise atrial flutter was eliminated after radiofrequency catheter ablation targeted at the IVCO-TA isthmus.

\section{Discussion}

Our study shows that the main electrophysiological determinant of the electrical induction of isthmus dependent atrial flutter in man is the slow conduction across the IVCO-TA-CSO isthmus. The presence of slow trans-isthmus conduction predisposes to local unidirectional block and, more importantly, determines the final induction of both counterclockwise and clockwise isthmus dependent atrial flutter. Otherwise, the antegrade or retrograde intraatrial and interatrial conduction characteristics do not differ between patients with or without atrial flutter.

SLOW CONDUCTION WITHIN THE ATRIAL FLUTTER CIRCUIT

An area of slow conduction is usually crucial for nearly all clinical reentrant tachyarrhythmias in human hearts, including atrial flutter. ${ }^{9}{ }^{10}$ Recent studies combining intra-atrial mapping and sequential testing by entrainment techniques $^{45}{ }^{16-18}$ have concluded that the circuit of typical atrial flutter is large and confined to the right atrium. The reentrant wavefront of atrial flutter circulates roughly parallel to the tricuspid annulus along the pathway built by natural right atrial barriers, including the crista terminalis, the eustachian ridge, the tricuspid annulus, and probably the tendon of Todaro. However, the existence of a local slow conduction within the atrial flutter circuit remains debatable. Based on entrainment studies at the high right atrium (with manifest entrainment) and the coronary sinus ostium (with concealed entrainment and short stimulus to flutter wave onset) regions, Olshansky et al and Feld et al suggested that an area of slow conduction exists between the LLRA and coronary sinus ostium at the low right atrium. ${ }^{9}{ }^{10}$ On the other hand, Olgin et al and Nakagawa et al both made a detailed, site specific analysis of local postpacing intervals and stimulus time to activation time difference during concealed entrainment and concluded that the IVCO-TA-CSO isthmus is a protected zone sandwiched between the tricuspid annulus and the crista terminalis and eustachian ridge, which prevents the leakage of activation current. ${ }^{45}$ An area of slow conduction was not particularly stressed. By direct pacing at the LLRA and CSO ends of the IVCO-TA-CSO isthmus, our study showed that the trans-isthmus conduction was abnormally slow (that is, $0.39-0.46 \mathrm{~m} / \mathrm{s}$ ) in patients with clinical or inducible atrial flutter, but nor$\mathrm{mal}$ in those without $(0.83-0.89 \mathrm{~m} / \mathrm{s})$ (table 2$)$. On the other hand, analysis of global atrial conduction characteristics including $\mathrm{P}$ wave duration, intra-atrial and interatrial antegrade conduction times, retrograde right atrial free wall and septal conduction velocities did not reveal any other slow conduction area in patients with or without atrial flutter. Our finding of a slow trans-isthmus conduction velocity in patients with isthmus dependent atrial flutter confirms the previous studies by Feld et $a l$ and Tai et al using a standard catheter pacing measurements in sinus rhythm, ${ }^{19}{ }^{20}$ and that by 
Shah et $a l{ }^{21}$ using a novel correlative three dimensional mapping technique in typical atrial flutter. The calculated intra-atrial conduction velocities in our atrial flutter patients were similar to those reported by Tai et al and Shah et al, with an estimated conduction velocity of $0.5-0.6 \mathrm{~m} / \mathrm{s}$ at the isthmus and $0.9-1.0$ $\mathrm{m} / \mathrm{s}$ in the septum and free wall of the right atrium during atrial pacing and during typical atrial flutter. However, in Tai's report, ${ }^{20}$ the slowing of isthmus conduction velocity was present in all patients, with or without atrial flutter. In Feld's report, ${ }^{19}$ the atrial conduction velocity was $0.50-0.57 \mathrm{~m} / \mathrm{s}$ at the right atrial free wall and septum in all the patients, regardless of the presence or absence of atrial flutter. However, the conduction velocity at the isthmus was $0.37-0.42 \mathrm{~m} / \mathrm{s}$ in atrial flutter patients, and remained $0.50-0.55 \mathrm{~m} / \mathrm{s}$ in patients without atrial flutter. Despite the variability in the measured conduction velocity in the right atrium, the demonstration of slow trans-isthmus conduction is quite consistent in all the studies. In addition, the abnormal isthmus conduction function predisposes to the occurrence of unidirectional block according to our study. The slow trans-isthmus conduction, the unidirectional trans-isthmus block, and the anatomical barriers in the right atrium may well lead to the initiation of counterclockwise or clockwise isthmus dependent atrial flutter, whenever atrial triggers occur. Among the prerequisites for reentry, ${ }^{22}$ slow conduction at the IVCO-TA-CSO isthmus appears to be the major determinant of electrophysiological initiation and maintenance of isthmus dependent atrial flutter in man.

ELECTRICAL INDUCTION OF ISTHMUS DEPENDENT ATRIAL FLUTTER

Olgin et al recently reported that pacing at the coronary sinus ostium would induce counterclockwise typical (isthmus dependent) atrial flutter, and LLRA pacing would induce clockwise flutter, following the creation of a unidirectional block at the IVCO-TA-CSO isthmus. ${ }^{23}$ Our study readily reproduced their observations and we share their conclusions about the site specificity for the induced atrial flutter activation patterns. However, a period of transient atrial tachycardia or atrial fibrillation was often noted in our study when counterclockwise or clockwise atrial flutter was about to be induced. In fact, two of the 24 counterclockwise atrial flutter episodes and six of the 17 clockwise type had had preceding atrial tachyarrhythmias before the final emergence of atrial flutter. Previous experimental work by Shimizu and colleagues ${ }^{24}$ and clinical report by Waldo and Cooper ${ }^{25}$ have emphasised the importance of a preceding period of atrial fibrillation or other arrhythmia in the "preparation" of the critical slow conduction and unidirectional block for the initiation of macro-reentrant atrial flutter. For the great majority of our patient population with a structurally normal heart, the contribution of transient atrial tachyarrhythmias to the initiation of isthmus dependent atrial flutter cannot be underestimated.
LIMITATIONS

First, without precise identification of intraatrial structure by intracardiac echocardiography ${ }^{4}$ or some other technique, the location of the LLRA and CSO ends of the IVCO-TA-CSO isthmus could not be confirmed with accuracy. In patients with inducible isthmus dependent atrial flutter, the exit of the isthmus could be functionally located by the demonstration of concealed entrainment with a short stimulus to flutter wave interval and a postpacing interval equal to the tachycardia cycle length. However, for the control group (that is, patients without atrial flutter), the LLRA and CSO sites could best be selected by multiple fluoroscopic views with angiographic verification, which might not be sufficiently reliable. Second, the calculation of atrial conduction velocity was focused on the IVCOTA-CSO isthmus and the right atrial wall mapped by the Halo catheter. Whether or not the slow conduction area in the low right atrium extended more laterally to the right atrial free wall or medially to the septum in some patients with clockwise or counterclockwise atrial flutter could not be confirmed. Nevertheless, the isthmus bordered by inferior vena caval orifice, the tricuspid annulus, and coronary sinus ostium was the common area of slow conduction for all the patients with isthmus dependent atrial flutter. Third, without comprehensive high density mapping of the atria, as shown in experimental studies, ${ }^{24}$ the importance of slow conduction in the IVCOTA-CSO isthmus could not be recognised or evaluated during the initiation of isthmus dependent atrial flutter by interposed atrial fibrillation. The period of chaotic fibrillatory rhythm might help stabilise the lines of functional block as well as the areas of slow conduction, which facilitated the final occurrence of macro-reentrant atrial flutter.

We thank Ms Yin-Hwa Lin and Ms Yueh-Ju Liao for their excellent secretarial assistance in the preparation of this manuscript. Presented in part at the 18th Annual Scientific Sessions of the North American Society of Pacing and Electrophysiology in New Orleans, Louisiana, USA, May 1997.

1 Waldo AL. Atrial flutter: new directions in management and mechanism. Circulation 1990;81:1142-3.

2 Olgin JE, Kalman JM, Lesh MD. Conduction barriers in human atrial flutter: correlation of electrophysiology and anatomy. F Cardiovasc Electrophysiol 1996;7:1112-16.

3 Cosio FG, Arribas F, Lopez GM, et al. Radiofrequency ablation of atrial flutter. F Cardiovasc Electrophysiol 1996;7: 60-70.

4 Olgin JE, Kalman JM, Fitzpatrick AP, et al. Role of right atrial endocardial structures as barriers to conduction during human type I atrial flutter: activation and entrainment mapping guided by intracardiac echocardiography. Circulamapping guided by intrac

5 Nakagawa H, Lazzara R, Khastgir T, et al. Role of the tricuspid annulus and the eustachian valve/ridge on atrial flutter: relevance of catheter ablation of the septal isthmus and a new technique for rapid identification of ablation success. Circulation 1996;94:407-24.

6 Kalman JM, Olgin JE, Saxon LA, et al. Activation and entrainment mapping defines the tricuspid annulus as the anterior barrier in typical atrial flutter. Circulation 1996;94: 398-406.

7 Caumechez B, Haissaguerre M, Fischer B, et al. Electrophysiologic effects of catheter ablation of inferior vena cava-tricuspid annulus isthmus in common atrial flutter. Circulation 1996;93:284-94.

8 Poty H, Saoudi N, Abdel Aziz A, et al. Radiofrequency catheter ablation of type I atrial flutter: prediction of late success by physiological criteria. Circulation 1995;92:138992 .

9 Olshansky B, Okumura K, Hess PG, et al. Demonstration of an area of slow conduction in human atrial flutter. $\mathcal{F} \mathrm{Am}$ Coll Cardiol 1990;16:1639-48. 
10 Feld GK, Fleck RP, Chen PS, et al. Radiofrequency catheter ablation for the treatment of human type 1 atrial flutter: identification of a critical zone in the reentrant circuit by endocardial

11 Waldo AL, Henthorn RW, Plumb VJ, et al. Demonstration of the mechanism of tion of ventricular tachycardia with rapid atrial pacing. $\mathcal{F}$ Am Coll Cardiol 1984;3:422-30.

12 Okumura K, Henthorn RW, Epstein AE, et al. Further observations on transient entrainment: importance of pacing site and properties of the components of the reentry circuit. Circulation 1985;72:1293-307.

13 Lesh MD, Kalman JM. To fumble flutter or tackle "Tach"? Toward updated classifiers for atrial tachyarrhythmias. $\mathcal{F}$ Cardiovasc Electrophysiol 1996;7:460-6.

14 Josephson ME. Atrial flutter and fibrillation. In: Clinical cardiac electrophysiology: techniques and interpretations.
Philadelphia: Lea and Febiger, 1993:275-310.

15 Surawicz B, Uhley H, Borum R. Optimal electrocardiography, Task Force I: Standardization of terminology and phy, Task Force I: Standardization of terminology and interpretation. B

16 Disertori M, Inama G, Vergara G, et al. Evidence of a reentry circuit in the common type of atrial flutter in man. Circulation 1983;67:434-40.

17 Della BP, Marenzi G, Tondo C, et al. Usefulness of excitable gap and pattern of resetting in atrial flutter for determining reentry circuit location. Am F Cardiol 1991;68:492-7.

18 Cosio FG, Lopez GM, Goicolea A, et al. Electrophysiologic

19 Feld GK, Mollerus M, Birgersdotter-Green U, et al. Conduction velocity in the tricuspid valve-inferior vena cava isthmus is slower in patients with type I atrial flutter compared to those without a history of atrial flutter. $7 \mathrm{Car}$ diovasc Electrophysiol 1997;8:1338-48.

20 Tai CT, Chen SA, Chiang CE, et al. Characterization of low right atrial isthmus as the slow conduction zone and pharmacological target in typical atrial flutter. Circulation 1997; 96:2601-11

21 Shah DC, Jaïs $\mathrm{P}$, Haïssaguerre $M$, et al. Three-dimensional mapping of the common atrial flutter circuit in the right atrium. Circulation 1997;96:3904-12.

22 Waldo A, Wit AL. Mechanisms of cardiac arrhythmias. Lancet 1993;341:1189-93.

23 Olgin JE, Kalman JM, Saxon LA, et al. Mechanism of initiation of atrial flutter in humans: site of unidirectional block and direction of rotation. $\mathcal{f}$ Am Coll Cardiol 1997;29:37684 .

24 Shimizu A, Nozaki A, Rudy Y, et al. Onset of induced atrial flutter in the canine pericarditis model. $\mathcal{F}$ Am Coll Cardiol 1991;17:1223-34.

25 Waldo AL, Cooper TB. Spontaneous onset of type I atrial flutter in patients. F Am Coll Cardiol 1996;28:707-12. 